\title{
Optimal Design and Power Allocation for Multicarrier Decode and Forward Relays
}

\author{
Ramy H. Gohary and Rozita Rashtchi and Halim Yanikomeroglu \\ Department of Systems and Computer Engineering, Carleton University, Ottawa, ON, Canada
}

\begin{abstract}
${ }^{1}$ Abstract-This paper considers a multicarrier communication system assisted by multiple relays, one for each subcarrier. The total power emitted by the source and the total power emitted by the relays are constrained to be less than the respective power budgets. The relays are assumed to operate in the full-duplex decode-and-forward mode, and the objective is to design the codebooks of the source and the relays jointly with the power allocations that maximize the total data rate that can be reliably decoded at the destination. To approach this goal, the design problem is cast as an optimization problem, which is unfortunately nonconvex and difficult to solve. The Karush-Kuhn-Tucker (KKT) system corresponding to this problem is analyzed, and despite the nonconvexity of the problem, we were able to use the KKT system to develop an efficient technique for solving it optimally.
\end{abstract}

Index Terms - decode-and-forward full-duplex relaying, optimization, KKT system, codebook correlation

\section{INTRODUCTION}

Communication-assisting nodes, known as relays, can effect a fundamental impact on the maximum data rate that can be reliably communicated between a transmitter-receiver pair. Relay operating modes are generally classified into either full-duplex or half-duplex ones. In the full-duplex mode, the relay transmits and receives information on the same physical channel, i.e., the same time slot and the same frequency. In contrast, in the half-duplex mode, transmission and reception takes places on orthogonal physical channels [1]. Halfduplex relays are more amenable to practical implementation, but the rates that they achieve are generally less than those achieved by their full-duplex counterparts. Advances in combining signal processing and beamforming techniques have been recently shown to successfully alleviate the difficulties that arise in the implementation of full-duplex relaying, and it is expected that with these advances, future wireless communication networks will rely more heavily on full-duplex rather than half-duplex relays [2].

The data rate that can be reliably communicated in the presence of a relaying node depends not only on the channel conditions, but also on the way in which the cooperation between the source, destination and relaying nodes is established. Various schemes are available in the literature including the amplify-and-forward (AF) [3], the compress-and-forward (CF), and the decode-and-forward (DF) relaying schemes [4]. The DF relaying scheme is known to achieve the capacity of a particular class of channels, that are referred to as being degraded [4], and generally outperforms the AF and $\mathrm{CF}$ relaying schemes when the source-relay link is substantially stronger than the source-destination link [1]. An extreme case is the one in which the source-destination link is severed, a situation in which the three-node cooperative channel reduces to a multihop one. In the latter case DF achieves capacity.

In DF, the relay decodes the codeword transmitted by the source and uses Wyner-Ziv binning [1] to determine the, so called, bin index

\footnotetext{
${ }^{1}$ This work is supported in part by the Natural Sciences and Engineering Research Council (NSERC) of Canada, in part by Huawei Canada Co., Ltd., and in part by the Ontario Ministry of Economic Development and Innovations ORF-RE (Ontario Research Fund-Research Excellence) program.
}

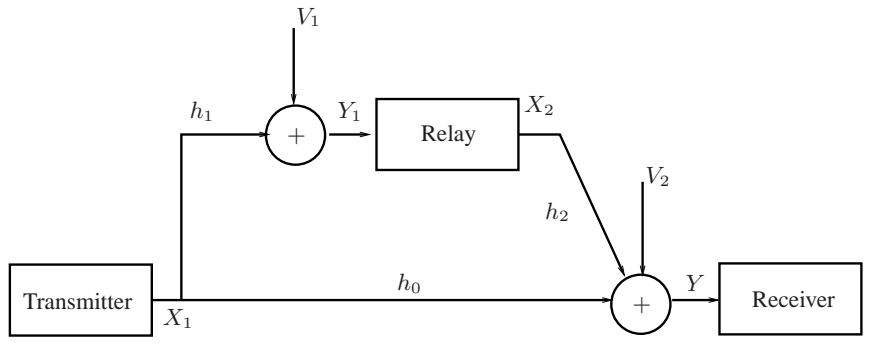

Fig. 1. Gaussian relay channel.

of that codeword. To establish cooperation, the relay transmits the codeword corresponding to the bin index to the destination, which combines this information with the information it obtained from the direct link to recover the source message. For scalar Gaussian relay channels, the DF optimal source and relay codebooks are Gaussian distributed with a particular correlation coefficient [5], [6], which must be optimized to maximize the rate that can be reliably communicated with the DF scheme.

In this paper we consider a multicarrier communication system assisted by multiple relays, one for each subcarrier. The total power emitted by the source and the total power emitted by the relays are constrained to be less than the respective power budgets. The relays are assumed to operate in the full-duplex DF mode, and the objective is to determine the optimal power allocation across the subcarriers at the source and the relays together with the optimal correlation coefficient between the source and relay codebooks on each subcarrier. To approach our goal, we begin in this paper by drawing insight from the DF relaying strategy in the single carrier case. Using this insight, we formulate the multicarrier design as an optimization problem, which is unfortunately nonconvex and difficult to solve. The Karush-Kuhn-Tucker (KKT) system corresponding to this problem is analyzed, and despite the nonconvexity of the problem, we were able to use the KKT system to develop an efficient technique for solving it optimally. Numerical results that illustrate the utility of the proposed technique are provided.

\section{DF RELAYING IN SINGLE-CARRIER COMMUNICATION SYSTEMS}

Consider the single carrier three-node Gaussian relay channel model in Figure 1. In this model the relay and destination received signals can be respectively expressed as

$$
Y_{1}=X_{1} h_{1}+V_{1}, \quad \text { and } \quad Y=X_{1} h_{0}+X_{2} h_{2}+V_{2},
$$

where $h_{i}$ are the complex gains of the links depicted in Figure 1, $i=0,1,2, X_{1}$ and $X_{2}$ are the source and the relay transmitted signals respectively, and $V_{1}$ and $V_{2}$ are the zero-mean unit variance additive Gaussian noise components at the relay and the destination, respectively. The signal, $X_{2}$, depends on the signal, $Y_{1}$, received by the relay in previous blocks, but not in the current one. It was 
shown in [5], [6] that the maximum data rate that can be reliably communicated by the DF scheme is achieved when $X_{1}$ and $X_{2}$ are Gaussian distributed with correlation coefficient $\theta \in[0,1]$, where

$$
\theta=\frac{\mathrm{E}^{2}\left\{X_{1} X_{2}\right\}}{\mathrm{E}\left\{X_{1}^{2}\right\} \mathrm{E}\left\{X_{2}^{2}\right\}}
$$

In other words, $X_{1}$ is related to $X_{2}$ via $X_{1}=\sqrt{\frac{\theta P_{1}}{P_{2}}} X_{2}+X_{10}$, where $P_{1}$ and $P_{2}$ are the transmit powers of the source and the relay, respectively, and $X_{10}$ is a Gaussian independent component with zero mean and variance $(1-\theta) P_{1}$.

For ease of exposition, let the absolute square channel gains of the source-destination, source-relay and relay-destination links be denoted by $g_{0}=\left|h_{0}\right|^{2}, g_{1}=\left|h_{1}\right|^{2}$ and $g_{2}=\left|h_{2}\right|^{2}$, respectively. Using this notation, the maximum rate that can be achieved by the DF relaying scheme is given by [1], [7]:

$$
\begin{array}{r}
\max _{0 \leq \theta \leq 1} \min \left\{\frac{1}{2} \log \left(1+g_{0} P_{1}+g_{2} P_{2}+2 \sqrt{\theta g_{0} g_{2} P_{1} P_{2}}\right),\right. \\
\left.\frac{1}{2} \log \left(1+(1-\theta) g_{1} P_{1}\right)\right\} .
\end{array}
$$

Our goal in this section is to establish relationships between the optimal correlation coefficient, $\theta$, and the transmit powers, $P_{1}$ and $P_{2}$. To begin with, we note that the first term in the minimization in (1) is monotonically increasing in $\theta$, and its minimum value is $\log \left(1+g_{0} P_{1}+g_{2} P_{2}\right)$. In contrast, the second term in the minimization in (1) is monotonically decreasing in $\theta$, and its minimum value is 0 . Hence, it can be readily seen that as $\theta$ decreases from 1 , the curve representing the second term is lower than that representing the first term. The two curves approach each other and the optimal value of $\theta$ is either the one at which the curves representing the two arguments intersect, or $\theta=0$, if these two curves do not intersect. To develop insight into the implications of each case, let us assume that these curves intersect at $\theta^{*}$. In this case, we have

$\log \left(1+g_{0} P_{1}+g_{2} P_{2}+2 \sqrt{\theta^{*} g_{0} g_{2} P_{1} P_{2}}\right)=\log \left(1+\left(1-\theta^{*}\right) g_{1} P_{1}\right)$.

To simplify notation, we will make the following definitions: $a \triangleq$ $\frac{g_{1}}{g_{0}}-1$ and $b=\frac{g_{2} P_{2}}{g_{0} P_{1}}$. Using this notation, the solution of (2) can be readily verified to be

$$
\sqrt{\theta^{*}}=\frac{\sqrt{a(a+1-b)}-\sqrt{b}}{a+1} .
$$

Solving (3) for $b$ yields

$$
\sqrt{b}=\sqrt{a\left(1-\theta^{*}\right)}-\sqrt{\theta^{*}} .
$$

We now make a few observations regarding the equalities in (3) and (4), which will later prove useful in studying the multicarrier case. First, we note that, with the source power, $P_{1}$, fixed, the relay transmit power, $P_{2}$, is monotonically decreasing with $\theta^{*}$. Second, we note from (3) that, for $\theta^{*}$ to be nonnegative, we must have $b \leq a$, which further implies that when the relay power is such that this inequality is violated, the curves representing the arguments of the minimization in (1) do not intersect and $\theta^{*}=0$. Third, we note from (4) that, for $\sqrt{b}$ to be nonnegative, we must have $\theta^{*} \leq \frac{a}{a+1}$. Fourth, we note that when $\theta^{*} \in\left[0, \frac{a}{a+1}\right]$, the rate achieved by the DF scheme can be expressed as $R=\frac{1}{2} \log \left(1+\left(1-\theta^{*}\right) g_{1} P_{1}\right)$. Finally, we note that if $a$ is less than 0, i.e., if $g_{1}<g_{0}$, the curves representing the two arguments of the minimization in (1) do not intersect. In fact, this situation corresponds to the case in which the received signal of the relay is weaker than that of the destination. In that case, the rate yielded by DF relaying is strictly less than that yielded by direct transmission, and it is more beneficial for the relay to be switched off. The achievable rate in that case is $\frac{1}{2} \log \left(1+g_{0} P_{1}\right)$.

\section{DF RELAYING IN MULTICARRIER COMMUNICATION SYSTEMS}

We now use the observations developed in Section II for the case of single carrier systems to analyze their multicarrier counterpart.

Consider a communication system operating over $N$ orthogonal carriers. The source and the relay are assumed to have total power budgets of $P_{T}$ and $P_{R}$, respectively. The relay uses the DF scheme on individual subcarriers, that is, on each subcarrier uses a particular correlation coefficient between the source and relay transmitted signal. However, the powers allocated to individual subcarriers are coupled by the overall power constraint at both the source and the relay. To characterize the maximum rate that can be achieved by this scheme, we denote the powers allocated by the source and the relay to subcarrier $i$ by $P_{1 i}$ and $P_{2 i}, i=1, \ldots, N$, respectively. The correlation coefficient for each subcarrier $i$ and the values corresponding to those defined in the single carrier case will be denoted by $\theta_{i}^{*}, a_{i}, b_{i}, g_{0 i}, g_{1 i}$ and $g_{2 i}, i=1, \ldots, N$.

Our goal now is to determine the power allocations, $\left\{P_{1 i}\right\}_{i=1}^{N}$ and $\left\{P_{2 i}\right\}_{i=1}^{N}$, and the correlation coefficients $\left\{\theta_{i}^{*}\right\}_{i=1}^{N}$ that maximize the sum of the rates that can be reliably communicated by the DF relaying scheme. Towards that end, we will find it convenient to define $\alpha_{i}=$ $1-\theta_{i}, i=1, \ldots, N$ to analyze the following optimization problem.

$$
\begin{aligned}
\max _{\left\{P_{1 i}\right\}_{i=1}^{N},\left\{P_{2 i}\right\}_{i=1}^{N},\left\{\alpha_{i}\right\}_{i=1}^{N}} & \frac{1}{2} \sum_{i=1}^{N} \log \left(1+\alpha_{i} g_{1 i} P_{1 i}\right), \\
\text { subject to } \quad & \sum_{i=1}^{N} P_{1 i} \leq P_{T}, \\
& \sum_{i=1}^{N} P_{2 i} \leq P_{R}, \\
& \alpha_{i} \leq 1, \quad i=1, \ldots, N, \\
& \sqrt{P_{2 i}} \geq \sqrt{\frac{g_{0 i} P_{1 i}}{g_{2 i}}}\left(\sqrt{a_{i} \alpha_{i}}-\sqrt{1-\alpha_{i}}\right), \\
&
\end{aligned}
$$

Before analyzing this problem, we make the following observations. First, the objective in (5a) is not concave and the constraints in (5e) are non-convex and subsequently the problem in (5) is non-convex and generally difficult to solve. Second, the objective in (5a) is monotonically increasing in $\alpha_{i}, i=1, \ldots, N$. Hence, including a nonnegativity constraint on $\left\{\alpha_{i}\right\}_{i=1}^{N}$ is not necessary. Finally, we have used the observation pointed out in Section II that $P_{2}$ is monotonically increasing in $\alpha$ to replace the equality in (4) with the respective inequality in (5e). Hence, for subcarriers for which this constraint is satisfied with equality the two arguments of the minimization in (1) are equal, and the optimal correlation coefficient is given by (3). For subcarriers for which (5e) is satisfied with strict inequality, the optimal correlation coefficient is 0 . Hence, it can seen that the formulation in (5) automatically captures the two possible types of subcarriers, those for which the curves representing the arguments of the minimization in (1) intersect and those for which the curve representing the second argument is strictly less than that representing the first one.

To analyze the problem in (5), we begin by writing the Lagrangian function

$$
\mathcal{L}=-\frac{1}{2} \sum_{i=1}^{N} \log \left(1+\alpha_{i} g_{1 i} P_{1 i}\right)+\lambda_{1}\left(\sum_{i=1}^{N} P_{1 i}-P_{T}\right)
$$




$$
\begin{gathered}
+\lambda_{2}\left(\sum_{i=1}^{N} P_{2 i}-P_{R}\right)+\sum_{i=1}^{N} \lambda_{3 i}\left(\alpha_{i}-1\right) \\
+\sum_{i=1}^{N} \lambda_{4 i}\left(\sqrt{\frac{g_{0 i}}{g_{2 i}}} \sqrt{P_{1 i}}\left(\sqrt{a_{i} \alpha_{i}}-\sqrt{1-\alpha_{i}}\right)-\sqrt{P_{2 i}}\right),
\end{gathered}
$$

where $\lambda_{1}$ and $\lambda_{2}$ are the Lagrange multipliers corresponding to the constraints in (5b) and (5c), respectively, and $\lambda_{3 i}$ and $\lambda_{4 i}$ are the Lagrange multipliers corresponding to the $i$-th constraints in $(5 \mathrm{~d})$ and (5e), respectively. Now, the KKT system that corresponds to the formulation in (5) can be expressed as follows:

$$
\begin{aligned}
& \sum_{i=1}^{N} P_{1 i}^{*} \leq P_{T}, \sum_{i=1}^{N} P_{2 i}^{*} \leq P_{R}, \alpha_{i} \leq 1, i=1, \ldots, N, \\
& \sqrt{P_{2 i}^{*}} \geq \sqrt{\frac{g_{0 i}}{g_{2 i}}} \sqrt{P_{1 i}^{*}}\left(\sqrt{a_{i} \alpha_{i}^{*}}-\sqrt{1-\alpha_{i}^{*}}\right), i=1, \ldots, N, \\
& \frac{\partial \mathcal{L}}{\partial P_{1 i}}=0, \quad \frac{\partial \mathcal{L}}{\partial P_{2 i}}=0, \quad \frac{\partial \mathcal{L}}{\partial \alpha_{i}}=0, \quad i=1, \ldots, N, \\
& \lambda_{1}, \lambda_{2}, \lambda_{3 i}, \lambda_{4 i} \geq 0, \quad i=1, \ldots, N, \\
& \lambda_{1}\left(\sum_{i=1}^{N} P_{1 i}^{*}-P_{T}\right)=0, \quad \lambda_{2}\left(\sum_{i=1}^{N} P_{2 i}^{*}-P_{R}\right)=0, \\
& \sum_{i=1}^{N} \lambda_{3 i}\left(\alpha_{i}^{*}-1\right)=0, \\
& \sum_{i=1}^{N} \lambda_{4 i}\left(\sqrt{\frac{g_{0 i}}{g_{2 i}}} \sqrt{P_{1 i}^{*}}\left(\sqrt{a_{i} \alpha_{i}^{*}}-\sqrt{1-\alpha_{i}^{*}}\right)-\sqrt{P_{2 i}^{*}}\right)=0 .
\end{aligned}
$$

The conditions in (9) yield

$$
\begin{aligned}
0 & =\frac{-\alpha_{i}^{*} g_{1 i}}{2\left(1+\alpha_{i}^{*} g_{1 i} P_{1 i}^{*}\right)}+\lambda_{1} \\
& +\frac{\lambda_{4 i}}{2} \sqrt{\frac{g_{0 i}}{g_{2 i}}}\left(\frac{\sqrt{a_{i} \alpha_{i}^{*}}-\sqrt{1-\alpha_{i}^{*}}}{\sqrt{P_{1 i}^{*}}}\right), \quad i=1, \ldots, N, \\
0 & =\lambda_{2}-\frac{\lambda_{4 i}}{2 \sqrt{P_{2 i}^{*}}}, \quad i=1, \ldots, N, \\
0 & =\frac{-P_{1 i}^{*} g_{1 i}}{2\left(1+\alpha_{i}^{*} g_{1 i} P_{1 i}^{*}\right)}+\lambda_{3 i}+\frac{\lambda_{4 i}}{2} \sqrt{\frac{g_{0 i}}{g_{2 i}}} \sqrt{P_{1 i}^{*}}\left(\sqrt{\frac{a_{i}}{\alpha_{i}^{*}}}\right. \\
& \left.\quad+\frac{1}{\sqrt{1-\alpha_{i}^{*}}}\right), \quad i=1, \ldots, N .
\end{aligned}
$$

Notice that, since the optimization problem in (5) is non-convex, the KKT conditions are not sufficient, but only necessary for optimality. However, by examining these conditions we will be able to develop an efficient method for solving (5).

Before examining the KKT conditions, we will distinguish between two classes of subcarriers: 1) subcarriers for which $a_{i} \leq 0$; and 2) subcarriers for which $a_{i}>0$. We will consider these cases separately.

\section{A. Subcarriers for which $a_{i} \leq 0$}

Let $\mathcal{I}_{0}$ be the set of subcarriers for which $a_{i} \leq 0$, i.e., those subcarriers for which $g_{0 i}>g_{1 i}$. In this case, the signal-to-noise ratio (SNR) of the signal observed by the relay is less than that observed by the destination, and the DF strategy cannot assist communication. In this case, it can be readily verified that it is better for the relay to be switched off, that is, it is better to set $P_{2 i}^{*}=0$ for $i \in \mathcal{I}_{0}$. Hence, for subcarriers in $\mathcal{I}_{0}$, the optimal power allocation is the water-filling one [8]. Using $\lambda_{0}$ to denote the optimal water level, we have

$$
P_{1 i}^{*}=\max \left\{\lambda_{0}-\frac{1}{g_{0 i}}, 0\right\}, \quad \forall i \in \mathcal{I}_{0},
$$

In the forthcoming discussion, we will focus on the case of $a_{i}>0$.

\section{B. Subcarriers for which $a_{i}>0$}

Referring to the case of a single carrier in Section II, it can be readily verified that for subcarriers with $a_{i}>0$, DF relaying is always beneficial and for each of those subcarriers the corresponding correlation coefficient $\theta_{i} \in\left[0, \frac{a_{i}}{1+a_{i}}\right]$. The class of subcarriers for which $a_{i}>0$ can be further divided into two subclasses, one in which the optimal correlation coefficient $\theta_{i}^{*}=0$ and one in which this coefficient is $\theta_{i}^{*}>0$. These subclasses will be addressed separately.

1) Subcarriers for which $a_{i}>0$ and $\theta_{i}^{*}=0$ : Let $\mathcal{I}_{1}$ be the set of subcarriers for which $a_{i}>0$ and the optimal correlation coefficients are $\theta_{i}^{*}=0$. For those subcarriers, $\alpha_{i}^{*}=1$ and the condition in (8) implies that $P_{2 i}^{*} \geq \frac{g_{1 i}-g_{0 i}}{g_{2 i}} P_{1 i}^{*}$. Since $P_{1 i}^{*}$ is finite, it follows from the condition in (16) that $\lambda_{4 i}=0$. Invoking these observations in the conditions in (15) and (14), respectively, yields $\lambda_{2}=0$ and

$$
P_{1 i}^{*}=\max \left\{\frac{1}{2 \lambda_{1}}-\frac{1}{g_{1 i}}, 0\right\}, \quad \forall i \in \mathcal{I}_{1} .
$$

Comparing (18) with (17), it can be seen that, in both $\mathcal{I}_{0}$ and $\mathcal{I}_{1}$, the optimal power allocation is a water-filling one. However, for the carriers in $\mathcal{I}_{0}$ the relay is off and water-filling is performed on the source-destination links, whereas for the carriers in $\mathcal{I}_{1}$, the relay is on and water-filling is performed on the source-relay links. The sum rate achieved on the carriers in $\mathcal{I}_{1}$ is given by $\sum_{i \in \mathcal{I}_{1}} \max \left\{\log \frac{g_{1 i}}{2 \lambda_{1}}, 0\right\}$.

It is worth noting that although this case has been addressed separately, the continuity of the constraints implies that it can be regarded as a limiting case for the one in which the optimal $\theta_{i}^{*}$ is strictly greater than 0 , which will be addressed next.

C. Subcarriers for which $a_{i}>0$ and $\theta_{i}^{*}>0$

Let $\mathcal{I}_{2}$ be the set of subcarriers for which $a_{i}>0$ and the optimal correlation coefficients satisfy $0<\theta_{i}^{*} \leq \frac{a_{i}}{a_{i}+1}$, i.e., $\alpha_{i}^{*} \in\left[\frac{1}{a_{i}+1}, 1\right)$.

Since for the subcarriers in $\mathcal{I}_{2}, \alpha_{i}^{*} \stackrel{<}{<}$, it follows from the condition in (12) that, for those subcarriers, $\lambda_{3 i}=0$. Using this and assuming that $P_{1 i}^{*}>0$ in the condition in (16), yields

$$
\lambda_{4 i}=g_{1 i} \sqrt{\frac{g_{2 i}}{g_{0 i}}} \frac{\sqrt{P_{1 i}^{*}}}{\left(1+g_{1 i} \alpha_{i}^{*} P_{1 i}^{*}\right)\left(\sqrt{\frac{a_{i}}{\alpha_{i}^{*}}}+\frac{1}{\sqrt{1-\alpha_{i}^{*}}}\right)}, \quad \forall i \in \mathcal{I}_{2} .
$$

Substituting for $\lambda_{4 i}$ from (19) into the conditions in (14) and (15) yields, respectively

$$
\begin{aligned}
& \lambda_{1}=\frac{g_{1 i} \sqrt{\alpha_{i}^{*}}}{2\left(1+g_{1 i} \alpha_{i}^{*} P_{1 i}^{*}\right)\left(\sqrt{\alpha_{i}^{*}}+\sqrt{a_{i}\left(1-\alpha_{i}^{*}\right)}\right)}, \quad \forall i \in \mathcal{I}_{2} . \\
& \lambda_{2}=\sqrt{\frac{g_{2 i}}{g_{0 i}}} \sqrt{\frac{P_{1 i}^{*}}{P_{2 i}^{*}}} \frac{g_{1 i}}{2\left(1+g_{1 i} \alpha_{i}^{*} P_{1 i}^{*}\right)\left(\sqrt{\frac{a_{i}}{\alpha_{i}^{*}}}+\frac{1}{\sqrt{1-\alpha_{i}^{*}}}\right)} \quad \forall i \in \mathcal{I}_{2} \text {. }
\end{aligned}
$$

From (20) and (21) we have

$$
\frac{\lambda_{2}}{\lambda_{1}}=\sqrt{\frac{g_{2 i} P_{1 i}}{g_{0 i} P_{2 i}}\left(1-\alpha_{i}^{*}\right)}, \quad \forall i \in \mathcal{I}_{2} .
$$

Now, since from (19), $\lambda_{4 i}>0$, it follows from (13) that (8) is satisfied with equality. Using this observation in (22) yields

$$
\sqrt{\frac{\alpha_{i}^{*}}{1-\alpha_{i}^{*}}}=\frac{1}{\sqrt{a_{i}}}\left(1+\frac{g_{2 i}}{g_{0 i}} \mu\right), \quad \forall i \in \mathcal{I}_{2},
$$

where $\mu=\frac{\lambda_{1}}{\lambda_{2}}$ is a subcarrier-independent constant.

Remark 1: If $\alpha_{i}^{*}=1$ for some subcarrier $i \in\{1, \ldots, N\}$, it follows from (19) that $\lambda_{4 i}=0$ and from (15) that $\lambda_{2}=0$ for all subcarriers. Hence, for any other subcarrier $i^{\prime} \in\{1, \ldots, N\}, \lambda_{4 i^{\prime}}=$ 
0 and (19) implies that either $\alpha_{i^{\prime}}^{*}=1$ or $P_{1 i^{\prime}}=0$, i.e., the source and relay codebooks on any used subcarrier are not correlated.

\section{An EFFICIENT Algorithm FOR OBTAINING OPTIMAL Correlation AND Power Allocation}

To develop an algorithm for obtaining the optimal $\left\{\theta_{i}^{*}\right\},\left\{P_{1 i}^{*}\right\}$ and $\left\{P_{2 i}^{*}\right\}$, we begin by assuming that the optimal $\mu=\frac{\lambda_{1}}{\lambda_{2}}$ is given. Such a $\mu$ exists only if $\theta_{i}^{*}>0$ (i.e., $\alpha_{i}^{*}<1$ ) for all $i \in\{1, \ldots, N\}$, cf. Remark 1. The optimal $\mu$ can be determined either by exhaustive search or by the method developed below. For now, we assume that the optimal $\mu$ and the set of subcarriers for which the optimal source transmit power is greater than zero are given. We will denote the latter set by $\mathcal{I}_{+} \subseteq \mathcal{I}_{2}$, and proceed as follows:

- For a given $\mu,(23)$ yields

$$
\alpha_{i}=\frac{\left(1+\frac{g_{2 i}}{g_{0 i}} \mu\right)^{2}}{a_{i}+\left(1+\frac{g_{2 i}}{g_{0 i}} \mu\right)^{2}},
$$

which for $a_{i} \geq 0$, implies that $\alpha_{i} \in\left[\frac{1}{1+a_{i}}, 1\right]$ is monotonically increasing in $\mu$.

- Using (20), the power allocated by the source to the $i$-th subcarrier can be expressed as

$$
P_{1 i}=\left[\frac{1}{2 \lambda_{1}\left(\alpha_{i}+\sqrt{a_{i} \alpha_{i}\left(1-\alpha_{i}\right)}\right)}-\frac{1}{g_{1 i} \alpha_{i}}\right]^{+} .
$$

This equation implies that for the source power on the $i$-th subcarrier to be greater than zero, we must have $2 \lambda_{1}\left(\alpha_{i}+\right.$ $\left.\sqrt{a_{i} \alpha_{i}\left(1-\alpha_{i}\right)}\right) \leq g_{1 i} \alpha_{i}$ for every $i \in \mathcal{I}_{+}$.

- Since $\lambda_{1}>0$, the KKT conditions imply that $\sum_{i=1}^{N} P_{1 i}=P_{T}$.

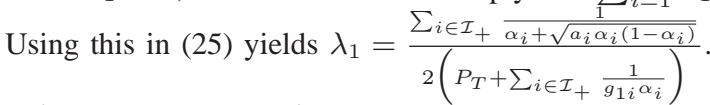

- Using (22), we can write

$$
\lambda_{2}^{2} P_{2 i}=\lambda_{1}^{2} \frac{g_{2 i}}{g_{0 i}} P_{1 i}\left(1-\alpha_{i}\right) .
$$

Since $\lambda_{2}>0$, the KKT conditions imply that $\sum_{i \in \mathcal{I}_{+}} P_{2 i}=P_{R}$, whence

$$
\lambda_{2}=\frac{\lambda_{1} \sqrt{\sum_{i \in \mathcal{I}_{+}} \frac{g_{2 i}}{g_{0 i}} P_{1 i}\left(1-\alpha_{i}\right)}}{\sqrt{P_{R}}}, \quad \forall i \in \mathcal{I}_{+} .
$$

Using this equality in (26) yields

$$
P_{2 i}=\frac{P_{R} P_{1 i} \frac{g_{2 i}}{g_{0 i}}\left(1-\alpha_{i}\right)}{\sum_{j=1}^{N} \frac{g_{2 j}}{g_{0 j}} P_{1 j}\left(1-\alpha_{j}\right)} \forall i \in \mathcal{I}_{+} .
$$

- To determine the optimal $\mu$ we note that $\mu=\frac{\lambda_{1}}{\lambda_{2}}$ must be nonnegative and must satisfy (27) when $P_{1 i}^{*}$ is given by (25) and $\alpha_{i}^{*}$ is given by (24). In other words, combining (27) with (25) and (24) yields that the optimal $\mu$ is a nonnegative root of the polynomial resulting from simplifying the following not particularly appealing equation:

$$
\begin{gathered}
P_{R}=\sum_{i \in \mathcal{I}_{+}} \frac{\frac{a_{i} g_{2 i}}{g_{0 i}} \mu^{2}}{a_{i}+\left(1+\frac{g_{2} i}{g_{0 i}} \mu\right)^{2}} \times \\
\left(\frac{P_{T}+\sum_{j \in \mathcal{I}_{+}} \frac{a_{j}+\left(1+\frac{g_{2 j}}{g_{0 j}} \mu\right)^{2}}{g_{1 j}\left(1+\frac{g_{2 j}}{g_{0 j}} \mu\right)^{2}}}{1+\sum_{\substack{j \in \mathcal{I}_{+} \\
j \neq i}} \frac{a_{j}+\left(1+\frac{g_{2 j}}{g_{0 j}} \mu\right)^{2}}{\left(1+\frac{g_{2 j}}{g_{0 j}} \mu\right)^{2}+a_{j}\left(1+\frac{g_{2 j}}{g_{0 j}} \mu\right)}}-\frac{a_{i}+\left(1+\frac{g_{2 i}}{g_{0 i}} \mu\right)^{2}}{g_{1 i}\left(1+\frac{g_{2 i}}{g_{0 i}} \mu\right)^{2}}\right) .
\end{gathered}
$$

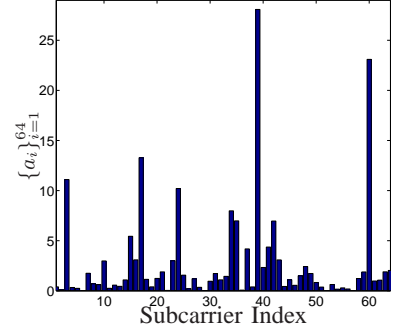

(a) $a_{i}, i=1, \ldots, 64$.

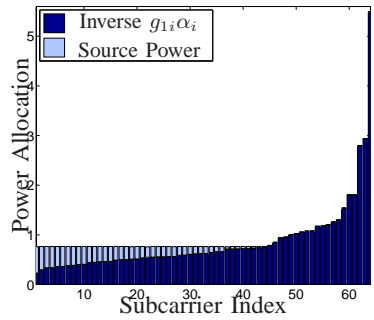

(c) Power allocation $\left(P_{R}=20 \mathrm{~dB}\right)$

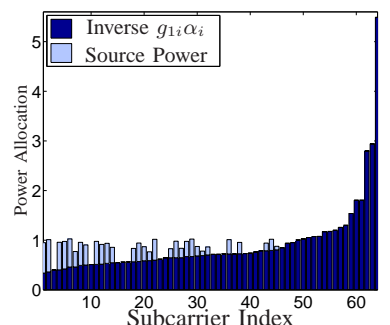

(b) Power allocation $\left(P_{R}=10 \mathrm{~dB}\right)$

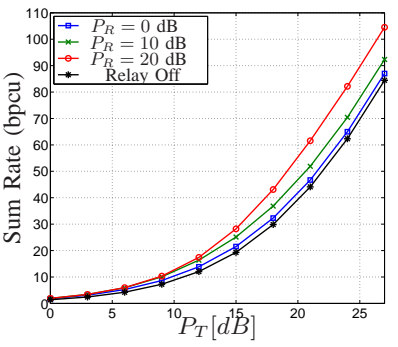

(d) Sum rate for various $P_{T}$ and $P_{R}$

Fig. 2. Optimal rates, correlation, and power allocation

- The optimal $\left\{\theta_{i}^{*}\right\},\left\{P_{1 i}^{*}\right\}$ and $\left\{P_{2 i}^{*}\right\}$ can be readily determined, by examining the values of $\mu$ that solve (28), and following the preceding steps starting form $\mathcal{I}_{+}=\{1, \ldots, N\}$, and discarding the subcarriers for which (25) yields $P_{1 i}=0$.

\section{NUMERICAL EXAMPLE}

For ease of exposition, we will restrict attention to the case in which $a_{i}>0, i=1, \ldots, N$. We consider a random instance with $N=64$ subcarriers and we use the algorithm outlined in Section IV to obtain the optimal values of $\left\{\alpha_{i}\right\}_{i=1}^{64},\left\{P_{1 i}\right\}_{i=1}^{64}$ and $\left\{P_{2 i}\right\}_{i=1}^{64}$. The values of $\left\{a_{i}\right\}$ in the considered instance are sorted according to the values of $\left\{\frac{1}{g_{1 i} \alpha_{i}}\right\}_{i=1}^{64}$ and plotted in Figure 2(a). The source powers $\left\{P_{1 i}\right\}_{i=1}^{64}$ and the sorted values of $\left\{\frac{1}{g_{1 i} \alpha_{i}}\right\}_{i=1}^{64}$ are depicted in Figures 2(b) and 2(c) for $P_{T}=10$ and $P_{R}$ equals 10 and $20 \mathrm{~dB}$, respectively. From these figures, it can be seen that, as expected from the analysis, increasing $P_{R}$ results in making the values of $\left\{\alpha_{i}\right\}$ approach 1 , and for the optimal power allocation to be the waterfilling one on $\left\{g_{1 i}\right\}$. (The darker bars in Figure 2(c) represent the inverse of $\left\{g_{1 i}\right\}$.) In Figure 2(d) we plot the sum rate achieved by DF relaying over all the subcarriers for various values of $P_{T}$ and $P_{R}$ when $\left\{a_{i}\right\}_{i=1}^{64}$ and $\left\{\frac{1}{g_{1 i}}\right\}_{i=1}^{64}$ as in Figures 2(a) and 2(c), respectively. For comparison, we also show the sum rate achieved by standard water-filling when the relay is off. This figure shows the substantial rate gain that can be achieved by increasing the power budget of the DF relay. For instance, for the scenario considered in this example, when the source and relay power budgets are $20 \mathrm{~dB}$, the rate gain introduced by the relay is in excess of 15 bits-per-channel-use (bpcu).

\section{CONCLUSION}

In this paper we analyzed the problem of joint optimization of the codebook design and power allocation for full-duplex multicarrier DF relaying. Despite the nonconvexity of this problem, we were able to use the KKT conditions to develop an efficient algorithm to solve it (28) and generate valuable insight into its structure. 


\section{REFERENCES}

[1] A. A. El Gamal, M. Mohseni, and S. Zahedi, "Bounds on capacity and minimum energy-per-bit for AWGN relay channels," IEEE Trans. Inf. Theory, vol. 52, pp. 1545-1561, Apr. 2006.

[2] Two-way (true full-duplex) wireless, (Toronto), June 2013.

[3] R. H. Gohary and H. Yanikomeroglu, "Joint optimization of the transmit covariance and relay precoder in general Gaussian amplify-and-forward relay channels," IEEE Trans. Inf. Theory, vol. 59, pp. 5331-5351, Sept. 2013

[4] T. M. Cover and A. A. El Gamal, "Capacity theorems for the relay channel," IEEE Trans. Inf. Theory, vol. IT-25, pp. 572-584, Sept. 1979.

[5] A. Høst-Madsen and J. Zhang, "Capacity bounds and power allocation for wireless relay channels," IEEE Trans. Inf. Theory, vol. 51, pp. 2020-2040, June 2005.

[6] B. Wang, J. Zhang, and A. Høst-Madsen, "On the capacity of MIMO relay channels," IEEE Trans. Inf. Theory, vol. 51, pp. 20-43, Jan. 2005.

[7] K. Luo, R. H. Gohary, and H. Yanikomeroglu, "Analysis of the generalized DF-CF for Gaussian relay channels: Decode or compress?," IEEE Trans. Commun., vol. 61, pp. 1810-1821, May 2013.

[8] T. M. Cover and J. A. Thomas, Elements of Information Theory. New York: Wiley, 1991. 